\title{
DCE and DW-MRI monitoring of vascular disruption following VEGF-Trap treatment of a rat glioma model
}

\author{
Benjamin A. Hoffa ${ }^{a}$ Mahaveer S. Bhojani ${ }^{b}$, John Rudge ${ }^{e}$, \\ Thomas L. Chenevert ${ }^{a}$, Charles R. Meyer ${ }^{a}$, Stefanie Galbán ${ }^{b}$, \\ Timothy D. Johnsond , Judith Sebolt Leopold ${ }^{a}$, Alnawaz Rehemtullab, \\ Brian D. Ross ${ }^{\mathrm{a}, \mathrm{c}}$ and Craig J. Galbán ${ }^{\mathrm{a} *}$
}

\begin{abstract}
Vascular-targeted therapies have shown promise as adjuvant cancer treatment. As these agents undergo clinical evaluation, sensitive imaging biomarkers are needed to assess drug target interaction and treatment response. In this study, dynamic contrast enhanced MRI (DCE-MRI) and diffusion-weighted MRI (DW-MRI) were evaluated for detecting response of intracerebral $9 \mathrm{~L}$ gliosarcomas to the antivascular agent VEGF-Trap, a fusion protein designed to bind all forms of Vascular Endothelial Growth Factor-A (VEGF-A) and Placental Growth Factor (PGF). Rats with 9 L tumors were treated twice weekly for two weeks with vehicle or VEGF-Trap. DCE- and DW-MRI were performed one day prior to treatment initiation and one day following each administered dose. Kinetic parameters ( $K^{\text {trans }}$, volume transfer constant; $k_{\mathrm{ep}}$, efflux rate constant from extravascular/extracellular space to plasma; and $\boldsymbol{v}_{\mathrm{p}}$, blood plasma volume fraction) and the apparent diffusion coefficient $(A D C)$ over the tumor volumes were compared between groups. A significant decrease in kinetic parameters was observed 24 hours following the first dose of VEGF-Trap in treated versus control animals $(p<0.05)$ and was accompanied by a decline in ADC values. In addition to the significant hemodynamic effect, VEGF-Trap treated animals exhibited significantly longer tumor doubling times $(p<0.05)$ compared to the controls. Histological findings were found to support imaging response metrics. In conclusion, kinetic MRI parameters and change in ADC have been found to serve as sensitive and early biomarkers of VEGF-Trap anti-vascular targeted therapy. Copyright $\odot 2011$ John Wiley \& Sons, Ltd.
\end{abstract}

Keywords: anti-angiogenic therapy; VEGF-Trap; glioma; DCE-MRI; DW-MRI; hemodynamics; diffusion; preclinical

\section{INTRODUCTION}

In Phase III clinical testing, Stupp and colleagues demonstrated the efficacy of concurrent temozolomide and radiation for treating newly diagnosed glioblastoma (1). With an improvement of median survival from 12 months to 14 months, this treatment regimen has become the standard of care. Although radiotherapy plus concomitant temozolomide provides a clinically meaningful and significant survival benefit, the prognosis remains poor for most patients with malignant gliomas. A number of molecularly targeted therapies are being investigated for their potential to significantly improve the outcome for these patients $(2,3)$.

Anti-angiogenic and antivascular therapies are at the forefront of development as viable treatment options for solid tumors $(4,5)$. Recent clinical trials have shown that such agents, e.g. Bevacizumab, provide improved efficacy for the treatment of recurrent brain tumors (6). The requirement of malignant gliomas for a continual supply of nutrients and oxygen provided by a vast network of newly forming intratumoral vessels, provides a sound scientific rationale for targeting tumor angiogenesis. Vascular Endothelial Growth Factor (VEGF) is one of the principal driving forces for tumors to maintain their highly proliferative potential. Elevated stimulation of angiogenesis through the production of VEGF occurs predominantly in high-grade tumors (7). Recent studies have also shown a significant reduction in both vascular
* Correspondence to: Craig J. Galbán, Ph.D., Assistant Professor, University of Michigan, Radiology Department, BSRB, Room D206, 109 Zina Pitcher Place, Ann Arbor, MI 48109-2200, USA.

E-mail: cgalban@med.umich.edu

a B. A. Hoff, T. L. Chenevert, C. R. Meyer, J. Sebolt Leopold, B. D. Ross, C. J. Galbán Department of Radiology, University of Michigan, Center for Molecular Imaging, Ann Arbor, MI, 48109, USA

b M. S. Bhojani, A. Rehemtulla

Department of Radiation Oncology, University of Michigan, Center for Molecular Imaging, Ann Arbor, Michigan, 48109, USA

C B. D. Ross

Department of Biological Chemistry, University of Michigan, Center for Molecular Imaging, Ann Arbor, Michigan, 48109, USA

d T. D. Johnson

Department of Biostatistics, University of Michigan, Center for Molecular Imaging, Ann Arbor, Michigan, 48109, USA

e J. Rudge

Regeneron Corporation, 777 Old Saw Mill Road, Tarrytown, NY, 10591, USA

Abbreviations used: VEGF-A, Vascular Endothelial Growth Factor-A; PGF, Placental Growth Factor; DCE-MRI, dynamic contrast enhanced magnetic resonance imaging; DSC, dynamic susceptibility contrast; DW-MRI, diffusion weighted MRI. 
permeability and neovascular formation in tumors treated with VEGF inhibitors (8-14) and have shown tumor regression in some cases (15). VEGF-Trap (Regeneron Pharmaceuticals, Inc., Tarrytown, NY), currently in clinical trials, is a decoy receptor protein effective in inhibiting VEGF signaling by binding with a high affinity to all isoforms of VEGF-A and placental growth factor (9-11,15-20). Preclinical studies have shown that this agent, when combined with standard treatments encompassing chemotherapy as well as radiotherapy, results in improved efficacy (21-23). Ultimately, these treatments are aimed at indirectly inhibiting tumor growth and possibly inducing cell death by limiting the availability of vital nutrients, which may improve the effectiveness of conventional therapies $(21,22)$.

Efforts are being made to evaluate imaging modalities to provide biomarkers of therapeutic-induced alterations in the tumor vasculature. Monitoring of volumetric changes prior to and following treatment initiation is the current standard of practice for assessing treatment effects. Although effective in predicting clinical outcome to therapy, prognosis may take 2-3 months following the start of treatment. Functional imaging complements traditional anatomical imaging for improved diagnosis and response assessment. Hemodynamic imaging techniques including dynamic contrast enhanced (DCE) and dynamic susceptibility contrast (DSC) MRI provide insights into tumor blood flow, blood volume and vessel permeability, which have shown promise as sensitive biomarkers of treatment-induced response (23). Most notably, DCE-MRI uses low contrast agent concentrations to produce signal enhancement, which can be tracked and fit to a pharmacokinetic model to extract such values as volume transfer constant $\left(K^{\text {trans }}\right)$, the flux rate constant between the extravascular extracellular space and plasma $\left(k_{\mathrm{ep}}\right)$, and blood plasma volume fraction $\left(v_{p}\right)$ (24-26). DCE-MRI has been used successfully to show decreased $K^{\text {trans }}$ in tumors very early after anti-VEGF treatments $(8,12,13)$. A decrease in $K^{\text {trans }}$ has been correlated with decreased growth rates and decreased levels of free VEGF, indicating effective drug targeting. The apparent diffusion coefficient (ADC), a quantitative measure of water mobility calculated from diffusion weighted (DW) MRI, has shown promise as a sensitive and reliable biomarker for cytotoxic therapies (27-30) that elicits a treatment-induced reduction in tumor cellularity (31). Increased cell death has been correlated with an increase in ADC. In this study, DCE- and DW-MRI were used to evaluate cellular and hemodynamic response of $9 \mathrm{~L}$ rat brain tumors to a VEGF-Trap antibody regimen.

\section{EXPERIMENTAL}

\section{Animal tumor models}

$9 \mathrm{~L}$ glioma cells were obtained from the Brain Tumor Research Center at the University of California at San Francisco. The cells were grown as monolayers in $10 \mathrm{~cm}^{2}$ sterile plastic flasks in Dulbecco's Modified Eagle Medium with 10\% fetal bovine serum, $100 \mathrm{IU} / \mathrm{mL}$ penicillin and $100 \mathrm{mg} / \mathrm{mL}$ streptomycin, and $2 \mathrm{mML}$-glutamine in a $37^{\circ} \mathrm{C}$ incubator. Prior to implantation, cells were harvested by trypsinization, counted, and re-suspended in serum-free medium for injection.

Tumor implantation was performed in 17 male Fischer-344 rats, 7 to 9 weeks old (Harlan Sprague-Dawley, Indianapolis, IN) weighing between 125 and $150 \mathrm{~g}$. Briefly, animals were anesthetized with a ketamine/xylazine mixture $(87 / 13 \mathrm{mg} / \mathrm{kg})$ administered intraperitoneally. A small incision was made over the right hemisphere of the cranium. A 1-mm diameter burr hole was drilled through the skull using a high-speed surgical drill, and a $5-\mu \mathrm{L}$ suspension containing $2 \times 10^{5} 9 \mathrm{~L}$ cells was slowly injected at a depth of $3 \mathrm{~mm}$. The burr hole was filled with bone wax, and the surgical area was cleaned using $70 \%$ ethanol. Vetbond ${ }^{\circledR}$ (3 M, St. Paul, MN) was used to close the incision until healed.

\section{Anti-angiogenic therapy}

Once tumor volumes reached $20-60 \mathrm{~mm}^{3}$ as determined using MRI, pre-treatment DCE and DW-MR images were acquired for all animals. Animals were then separated into two groups and were treated with either $25 \mathrm{mg} / \mathrm{kg}$ VEGF-Trap $(n=10)$ or $12.5 \mathrm{mg} / \mathrm{kg}$ human Fc (vehicle protein) $(n=7)$. Treatments were administered subcutaneously twice weekly for two weeks (Fig. 1A).

\section{MRI scans}

MRI scans were performed on a $9.4 \mathrm{~T}, 16 \mathrm{~cm}$ horizontal bore Varian (Palo Alto, CA) DirectDrive system with a quadrature rat head coil (Doty Scientific, Inc., Columbia, SC). During all MRI procedures, animals were anesthetized with a 1-2\% isofluorane/air mixture and body temperature was maintained using a heated air system (Air-Therm, World Precision Instruments, Sarasota, $\mathrm{FL})$. MR images were acquired $24 \mathrm{~h}$ prior to treatment initiation and $24 \mathrm{~h}$ after each treatment (Fig. 1A). Each MR experiment consisted of a fast spin-echo-based $T_{1}$-mapping sequence and DCE- and DW-MRI sequences with a total overall acquisition time for each imaging session of approximately $45 \mathrm{~min}$.

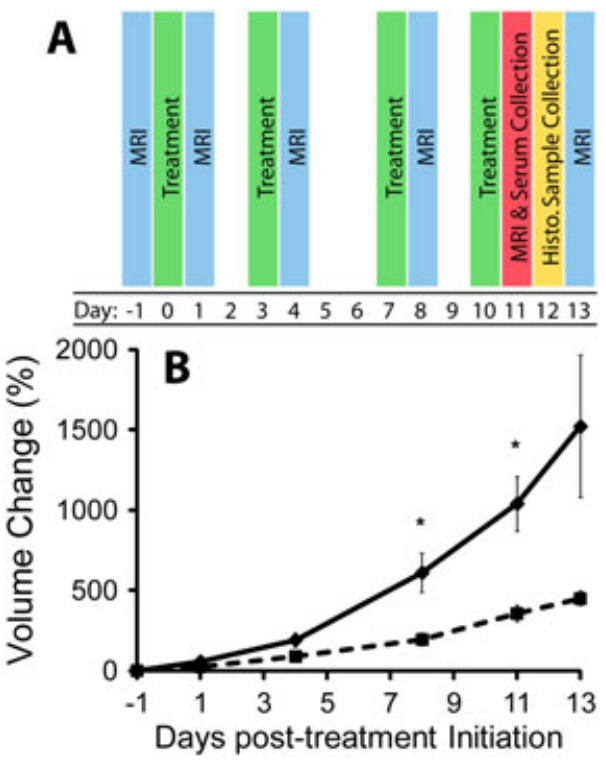

Figure 1. Diagrammatic presentation of study time points (A). Treatments are highlighted in green, MRI (both DCE and DWI) are highlighted blue, MRI and blood serum collection were performed on Day 11 (red), and histological samples were taken at approximately Day 12 (yellow). Plot showing relative change in tumor volume in control (diamond, solid line) and treated (square, dotted line) groups over the study time period (B). Treated animals generally showed a significant slowing of tumor growth compared to controls. Doubling times in the control and treated groups were $3.76( \pm 0.325)$ and 5.32 ( \pm 0.319) days, respectively $(p=0.004)$. Significance in relative change in volume between groups occurred at days 8 and 11 post-therapy. Data are presented as means $\pm S E M$. Significance was assessed at $p<0.05$ and indicated by *. 
DW-MRI was performed using a spin-echo sequence with a navigator echo for motion correction and gradient waveforms sensitive to isotropic diffusion (32). Images were acquired using the following parameters: repetition time/echo time $(T R / T E)=$ $4000 / 47 \mathrm{~ms}$, field of view $(\mathrm{FOV})=30 \mathrm{~mm}$, matrix size $=128 \times 64$, slice thickness $=1 \mathrm{~mm}$, slice number $=13$, and $b$-values (diffusion weighting) of 120 and $1200 \mathrm{~s} / \mathrm{mm}^{2}$.

DCE-MRI was performed using a $T_{1}$-weighted gradient-echo sequence with the following parameters: TR/TE $=85 / 3.2 \mathrm{~ms}$, flip angle $=20^{\circ}, F O V=30 \mathrm{~mm}$, matrix size $=128 \times 64$, slice thickness $=1 \mathrm{~mm}$, slice number $=13$ and averages $=2$. Image datasets were acquired over a 15-min period with a time resolution of 10.9 s. Following 1 min of scanning ( 6 images), a bolus dose of gadolinium-DTPA (Magnevist, Bayer Healthcare Pharmaceuticals; $0.15 \mathrm{mmol} / \mathrm{kg}$, diluted $1: 8.3$ from $0.5 \mathrm{mmol} / \mathrm{mL}$ in $0.9 \%$ saline solution to $0.06 \mathrm{mmol} / \mathrm{ml}$ ) was administered via tail-vein catheter at a rate of $4 \mathrm{~mL} / \mathrm{min}$.

\section{Image reconstruction and analysis}

Tumors were manually contoured on the low- $b$ images from the DW-MRI sequence $\left(b=120 \mathrm{~s} / \mathrm{mm}^{2}\right)$. These volumes of interest (VOI) were used to determine tumor volume and whole-tumor mean values generated from quantification of DW-MRI and DCE-MRI data. Tumor doubling times were calculated for each animal from exponential fits in Excel (Microsoft, Redmond, WA) to each individual growth time course (33).

Apparent diffusion coefficients were calculated from the two diffusion weightings ( $b$-values) using the following equation:

$$
A D C_{1-2}=\ln \left(\frac{S_{1}}{S_{2}}\right) /\left(b_{2}-b_{1}\right)
$$

where $S_{1}$ and $S_{2}$ are the signal intensities at $b$-values $b_{1}=120 \mathrm{~s} / \mathrm{mm}^{2}$ and $b_{2}=1200 \mathrm{~s} / \mathrm{mm}^{2}$, respectively, and $A D C_{1-2}$ is the apparent diffusion coefficient obtained using $b_{1}$ and $b_{2}$.

Tumor kinetic parameters were determined by a voxel-wise three-parameter fit on the acquired time-resolved $T_{1}$-weighted images using a tri-exponential arterial input function (AIF) for blood plasma concentration $\left(C_{p}\right)(12)$ :

$$
C_{p}(t)=A 1 \cdot \exp \left(-\alpha_{1} \cdot t\right)+A 2 \cdot \exp \left(-\alpha_{2} \cdot t\right)+A 3 \cdot \exp \left(-\alpha_{3} \cdot t\right)
$$

with $\mathrm{A} 1=0.8259, \quad \mathrm{~A} 2=0.2230, \mathrm{~A} 3=0.1565 \mathrm{mM}, \quad \alpha_{1}=1.220$, $\alpha_{2}=0.156$, and $\alpha_{3}=0.017 / \mathrm{min}$. In this study, we followed the referenced contrast injection procedure used for this AIF and assumed negligible differences in AIF over time as well as between animals. This AIF was incorporated into a generalized kinetic model, equivalent to the efflux-corrected Patlak model $(34,35)$ :

$$
C_{t}(t)=K^{\text {trans }} \int_{0}^{t} C_{p}(\tau) \cdot \exp \left(-k_{e p}(t-\tau)\right) d \tau+v_{p} C_{p}(t)
$$

where $K^{\text {trans }}$ is the volume transfer constant, $k_{\mathrm{ep}}$ is the flux rate constant between extravascular extracellular space (EES) and plasma, and $v_{p}$ is the blood plasma volume fraction. Fits were performed using a non-linear least-squares algorithm, and goodness-of-fit (GoF) was monitored to confirm reliable results; GoF was defined as the normalized root mean square error. Baseline signal intensity was calculated as a mean from the first $\sim 6$ images before contrast injection excluding the first image due to non-steady-state signal. Tissue concentrations of contrast agent were determined using the following equation: $1 / T_{1}=R^{*}[\mathrm{Gd}-\mathrm{DTPA}]+1 / T_{10}$. The relaxivity
(R) was determined to be $5.5 \mathrm{ml} /(\mathrm{mmol} \mathrm{s})$ by acquiring $T_{1}$ maps of $1 \mathrm{~cm}^{3}$ syringes filled with saline $(0.5 \mathrm{M})$ and $13 \mathrm{Gd}$-DTPA concentrations ranging from 0 to $10 \mathrm{mM}$. The relaxation constant was assumed constant between animals and time points. $T_{10}$ is the $T_{1}$ of the tumor tissue prior to injection of contrast agent. $T_{10}$ was set to $2.5 \mathrm{~s}$ which was the average of $T_{1}$ measurements obtained in each animal pre-therapy using a fast spin-echo sequence with the following parameters: five TR values of 5, 1.5, 1, 0.8 and $0.6 \mathrm{~s}$, with 1, 1, 1, 2, and 4 averages, respectively; effective echo time $(T E)=39.68 \mathrm{~ms}$, echo train length $(E T L)=4,2$ dummy scans, matrix size $=128 \times 256, F O V=3 \mathrm{~cm}$, slice thickness $=1 \mathrm{~mm}$. Parameter maps of $K^{\text {trans }}, k_{\mathrm{ep}}, v_{\mathrm{p}}$, and GoF were saved for the tumor VOI. Non-enhancing tumor tissue within the VOI was excluded from the analysis due to the model's inability to accurately describe the tissue's kinetic properties. Therefore, voxels with lower than $0.35 \mathrm{GoF}$ or $0.002 v_{\mathrm{p}}$ were excluded in mean tumor measurements. All reconstruction and analyses were performed using in-house programs written in MATLAB (The Mathworks, Natick, MA, USA). Parameter maps shown in Figure 2 were interpolated to $256 \times 256$ matrix size for display only.

\section{Histology}

Three representative animals were randomly selected from each group for tumor histology at approximately 12 days post-treatment initiation (Fig. 1A). 9L tumors from control and treated animals were placed in buffered formalin overnight, dehydrated in $70 \%$ ethanol, and subsequently embedded in paraffin. Tissue sections were prepared for histological processing by routine techniques. Briefly, paraffin sections ( $5 \mu \mathrm{m}$ thick) were cut on a microtome and heated for $20 \mathrm{~min}$ at $65^{\circ} \mathrm{C}$. Slides were de-paraffinized in xylene with three changes for $5 \mathrm{~min}$ each, then rehydrated through an alcohol gradient for 2 min each (100\% alcohol, 95\% alcohol, 70\% alcohol). Some sections were first stained using a Gill's $2 X$ hematoxylin solution followed by eosin, while others were stained using the rabbit polyclonal antibody to Von Willebrand Factor III (vWf) to highlight tissue vasculature, and counterstained for the nuclei. For counting tumor vasculature, representative fields were obtained from the vWf-stained slides at random on each of three controls and three treated tumor slices. Brown-stained areas greater than about $10 \mu \mathrm{m}$ in diameter were considered positive stains for the purpose of counting. Positive stains were counted using IMAGEJ software $(\mathrm{NIH})$ and used to quantify the difference between groups. On additional sections, ApopTag or Ki-67 stains were performed using standard techniques to highlight apoptotic or proliferating cells in the tumor, respectively.

\section{Assessment of free and bound VEGF}

Blood serum samples were collected through a tail-vein catheter $24 \mathrm{~h}$ after the final dose of VEGF-Trap $(n=7)$ or vehicle $(n=3)$ (Fig. 1A). Samples were stored short-term in a $3{ }^{\circ} \mathrm{C}$ refrigerator until being shipped on ice to Regeneron Pharmaceuticals for analysis.

Free VEGF trap was measured using a sandwich ELISA method in which hVEGF165 (Regeneron Pharmaceuticals, Inc) diluted in $0.05 \mathrm{M}$ carbonate-bicarbonate buffer was used as the capture protein at a concentration of $2 \mu \mathrm{g} / \mathrm{mL}$. The antigen was VEGFTrap (Regeneron Pharmaceuticals, Inc.) prepared in 1\% BSA (KP Labs). Samples and standard were incubated for $2 \mathrm{~h}$ at room temperature and the excess was removed in subsequent washes. The bound material was then reported with a mouse monoclonal antibody (P10 G1F6, Regeneron Pharmaceuticals, Inc) then 
A

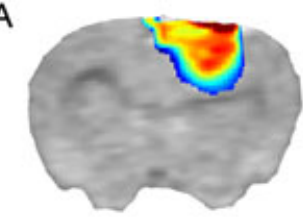

B

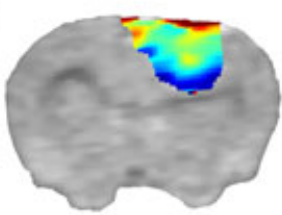

C

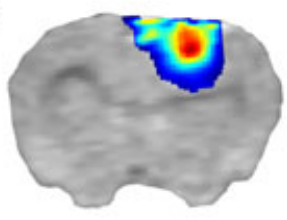

D

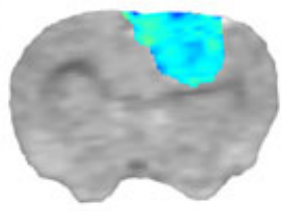

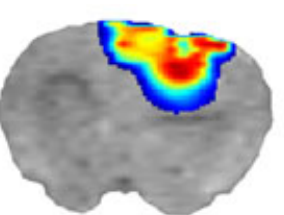
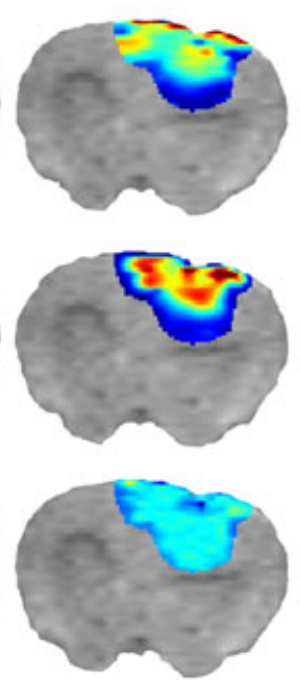

E

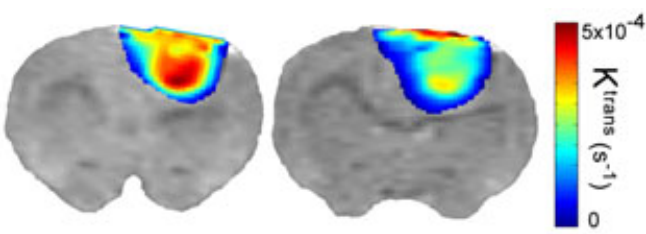

F

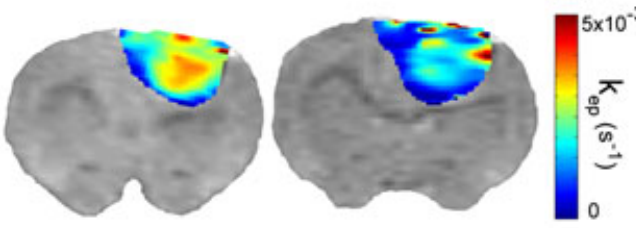

G

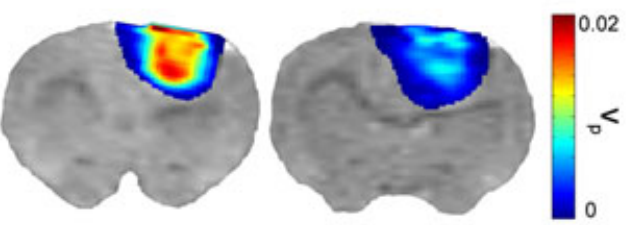

H

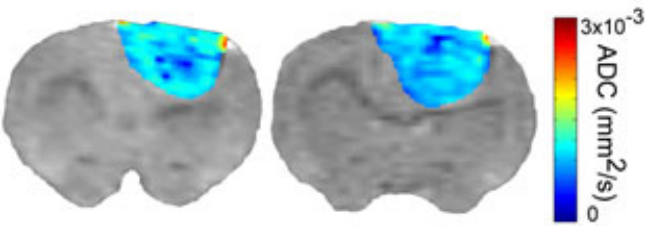

Figure 2. $T_{2}$-weighted images with color overlays of parametric maps are shown for a representative animal in the control group (A-D) and the VEGFTrap-treated group (E-H) prior-to (Day -1, left image) and following (Day 1, right image) the initial treatment. The initial drop for the VEGF-Trap-treated group in $K^{\text {trans }}(-27 \%), k_{\mathrm{ep}}(-12 \%)$, and $v_{\mathrm{p}}(-64 \%)$ can clearly be seen here $(\mathrm{E}-\mathrm{G})$. ADC shows a small, but significant drop ( $\left.-7 \%\right)$ by the first day post-therapy (H). Tumor heterogeneity was observed at individual time points. Nevertheless response to VEGF-Trap did not vary spatially throughout the tumor.

a secondary antibody was used. Anti-mouse HRP (Jackson Laboratories) TMB substrate (Sigma, T8665) was used in the color development, the plates were read at $450-570 \mathrm{~nm}$ and results analyzed using the SoftMax Pro 5.3 (Molecular Devices).

Bound endogenous rat VEGF was determined by an ELISA developed using the antibody to Rat VEGF164 affinity purified polyclonal antibody (R\&D Systems, Cat\#AF564) as the capture antibody. The antigen was prepared fresh with rVEGF bound to two molar quantities of the VEGF-Trap and allowed to incubate at room temperature for $1 \mathrm{~h}$. This complex was used to generate a standard curve. Samples were bound to the plate, and after washing away the unbound rVEGF the unknown and controls were reported with an anti-human Fc IgG (Sigma, A-0170).

The captured protein used in this assay was recombinant rat VEGF at $2 \mu \mathrm{g} / \mathrm{mL}$ in $0.05 \mathrm{~m}$ carbonate-bicarbonate buffer. The antigen used was the mouse monoclonal antibody P10 (Regeneron Pharmaceuticals, Inc), which was reported using a goat anti-mouse antibody (Jackson Laboratories) and the samples were reported with an anti-rat antibody (Promega). The samples were diluted 1:1000 to dilute out the serum effects of this assay.

\section{Statistics}

A paired Student's $t$-test was used to compare the percent change of parameter values between pre- and post-therapy time points for each animal. An unpaired Student's $t$-test was used to perform group comparisons in percent change of parameter values at each time point and tumor doubling times. Results were declared statistically significant at the two-sided 5\% comparison-wise significance level $(p<0.05)$. Values are given as means \pm SEM.

\section{RESULTS}

Therapeutic intervention was initiated at tumor volumes of $35.8 \pm 5.0 \mathrm{~mm}^{3}$ and $36.4 \pm 8.6 \mathrm{~mm}^{3}$ for VEGF-Trap therapy and vehicle-treated control animals, respectively. Based on ELISA analyses, VEGF-Trap proved effective at binding to virtually all endogenous VEGF with no detectable VEGF and excess VEGF-Trap within the blood samples tested (Table 1). Data for the control group were not presented due to the absence of VEGF-Trap and VEGF

Table 1. ELISA analysis of endogenous VEGF

\begin{tabular}{|c|c|c|c|}
\hline & Free VEGF-Trap & Bound VEGF & Free Rat VEGF \\
\hline VEGF-Trap & $98660 \pm 11529$ & $1540.45 \pm 149.30$ & $0.00 \pm 0.00$ \\
\hline BLQ & 359 & 309 & 783 \\
\hline \multicolumn{4}{|c|}{$\begin{array}{l}\text { ELISA analysis was performed to assess the effectiveness of VEGF-Trap in attenuating the signaling of free rat VEGF. Blood serum } \\
\text { levels were measured on Day } 11 \text { post-treatment. Free VEGF-Trap, unbound VEGF-Trap; Bound VEGF, VEGF/VEGF-Trap complex; } \\
\text { Free Rat VEGF, unbound endogenous VEGF; BLQ, Below Level of Quantification. Quantities are shown as group mean } \pm \text { SE } \\
(n=7) \text {. All units are ng/mL. VEGF levels for control animals were immeasurable and are not shown. }\end{array}$} \\
\hline
\end{tabular}


levels being below quantification. Low levels of endogenous VEGF in non-VEGF-Trap treated animals have been reported in previous studies $(19,36)$. As shown in Figure 1B, inhibition of VEGF signaling within the tumor resulted in significantly larger percentage changes in control tumor volumes at 8 and 11 days post-treatment initiation than those observed for VEGF-Trap treated tumors $(p<0.05)$. Tumor volume doubling times were also affected as evidenced by values of $3.76( \pm 0.325)$ and 5.32 ( \pm 0.319$)$ days for vehicle and VEGF-Trap groups, respectively $(p=0.004)$.

Representative vascular kinetic and diffusion parameter maps are shown as color overlays in Figure 2. Partial-voluming effects are apparent in Figure 2, evidenced by reduced kinetic measurements on the periphery of the color overlays. In vehicle-treated animals, negligible changes in parameter values were observed one day post-treatment initiation compared to the pre-treatment baseline (Fig. 2A-D). In striking contrast, a single treatment with VEGF-Trap resulted in a substantial decrease in all kinetic parameters (Fig. 2E-H) consistent with successful drug targeting. In general, all kinetic parameters showed spatial variability within the $9 \mathrm{~L}$ tumors at any given time point. Nevertheless, a spatially uniform response to VEGF-Trap was observed throughout the tumor for all kinetic metrics.

The two kinetic transfer constants, $K^{\text {trans }}$ and $k_{\mathrm{ep}}$, and blood volume fraction $\left(v_{p}\right)$ were found to be dependent on VEGF-Trap treatment, again consistent with strong effectiveness in target modulation by the drug (Fig. 3). The reduction in $K^{\text {trans }}$ was observed following the first day of treatment, with a significant difference in $K^{\text {trans }}(27 \pm 3.1 \%, p=0.002)$ when compared to vehicle-treated animals. Similar trends were observed for $v_{p}$ with a change of $63 \pm 2.3 \%(p<0.001)$. Subsequent to the initial decrease, parameter values plateaued and remained stable throughout the rest of the treatment cycle. A gradual reduction in $k_{\mathrm{ep}}$ resulted in significant differences between the two treatment groups by Day 3 post-treatment initiation (Fig. 3). The maximum change in $k_{\mathrm{ep}}(34.6 \pm 5.8 \%, p=0.007)$ was observed at Day 11.

In line with the kinetic parameters, ADC values declined following VEGF-Trap treatment (Fig. 3). Although not as pronounced as the changes in kinetic metrics, a significant reduction in the change in ADC values was observed in VEGF-Trap treated tumors when compared to controls for all time points after treatment. $A D C$ values did not reach a minimum value until Day 8 with a percentage decrease of $17.8 \pm 3.2 \%(p=0.027)$. Although ADC values for VEGF-Trap animals increased slightly from Day 8 to 11 , this change was not significant.

Histological sections of representative animals from control $(n=3)$ and treated $(n=3)$ groups taken 24 hours after the last treatment (11 days post-initial-treatment) were subjected to $\mathrm{H} \& \mathrm{E}, \mathrm{Ki}-67$, Von Willebrand / Factor VIII (vWF) and ApopTag staining. As observed in Figure 4, a 14\% reduction in Ki-67 staining was observed for VEGF-Trap treated tumors. This difference, although consistent with reduced proliferative potential, was not significant $(p=0.25)$. In contrast, vWF stained sections revealed that vessel numbers were significantly lower in VEGFTrap-treated versus vehicle-treated animals ( $p=0.011$; Fig. 5 ). Interestingly, ApopTag staining revealed that apoptosis appeared to be localized within the endothelial cells of the vasculature, as shown by heavily stained regions in Figure 6 . In contrast, tumor tissue did not show significant staining, and was therefore not quantified and compared between groups. The results observed in Figure 6 are consistent with the loss in vessel number (Fig. 5) as well the reduction in MRI-determined kinetic measures (Fig. 3). However, loss of vasculature due to VEGF

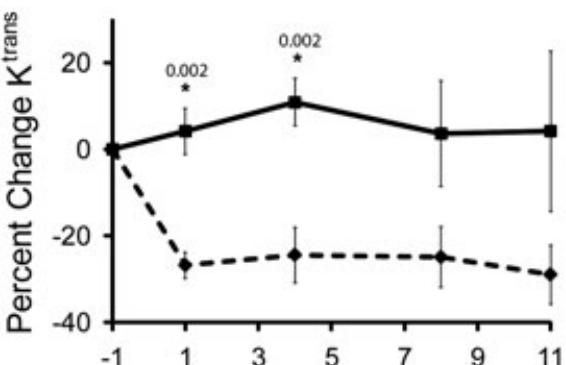

Days post-treatment Initiation

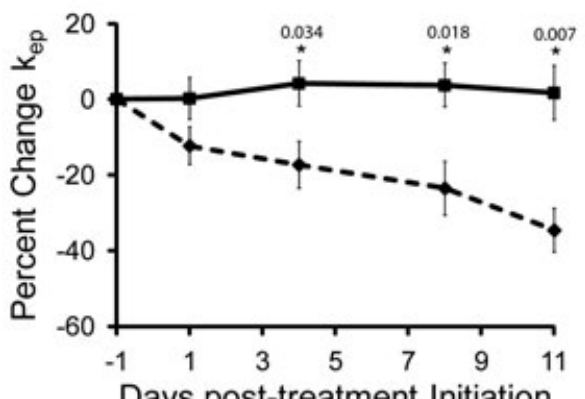

Days post-treatment Initiation

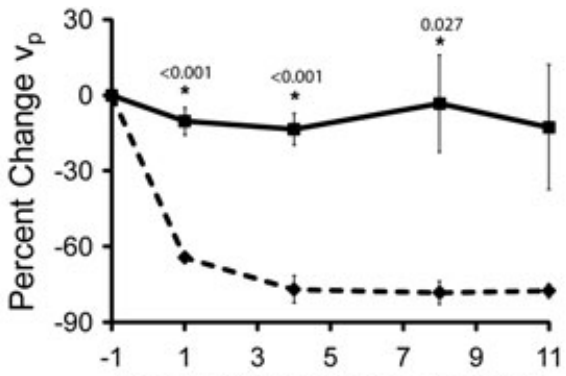

Days post-treatment Initiation

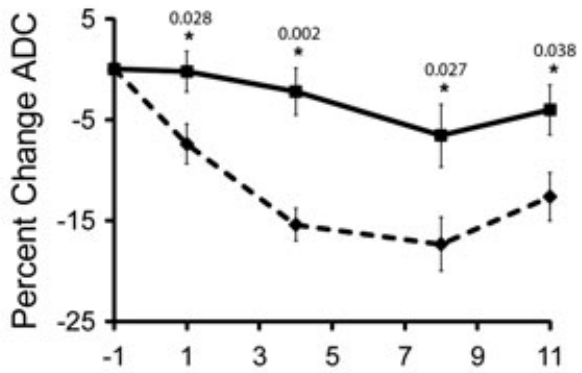

Days post-treatment Initiation

Figure 3. Plots of relative change in kinetic and diffusion parameters for the treated group (diamond, dotted line) shown together with the control (square, solid line). A significant decrease in $K^{\text {trans }}$ and $v_{p}$ occurred on the first day post-therapy and persisted throughout the study. Tumor ADC steadily decreased in VEGF-Trap treated tumors up to $15 \%$ from the initial value. In contrast, $k_{\mathrm{ep}}$ continued to decrease throughout the study. Data are presented as means \pm SEM. Significance was assessed at $p<0.05$ and indicated by * under their respective $p$-values. Baseline parameter values for vehicle and VEGF-Trap treated animals are: for $K^{\text {trans }}, 2.4 \pm 0.1 \times 10^{-4} / \mathrm{s}$ and $2.3 \pm 0.1 \times 10^{-4} / \mathrm{s}(p=0.7)$, respectively; $v_{p}, 7.6 \pm 1.3 \times 10^{-3}$ and $8.1 \pm 0.5 \times 10^{-3}$ $(p=0.7)$, respectively; $k_{\mathrm{ep}}, 1.9 \pm 0.1 \times 10^{-3}$ and $1.9 \pm 0.1 \times 10^{-3}(p=0.8)$, respectively; and ADC, $1.1 \pm 0.02 \times 10^{-3} \mathrm{~mm}^{2} / \mathrm{s}$ and $1.0 \pm 0.02 \times 10^{-3} \mathrm{~mm}^{2} / \mathrm{s}$ $(p=0.1)$, respectively. 

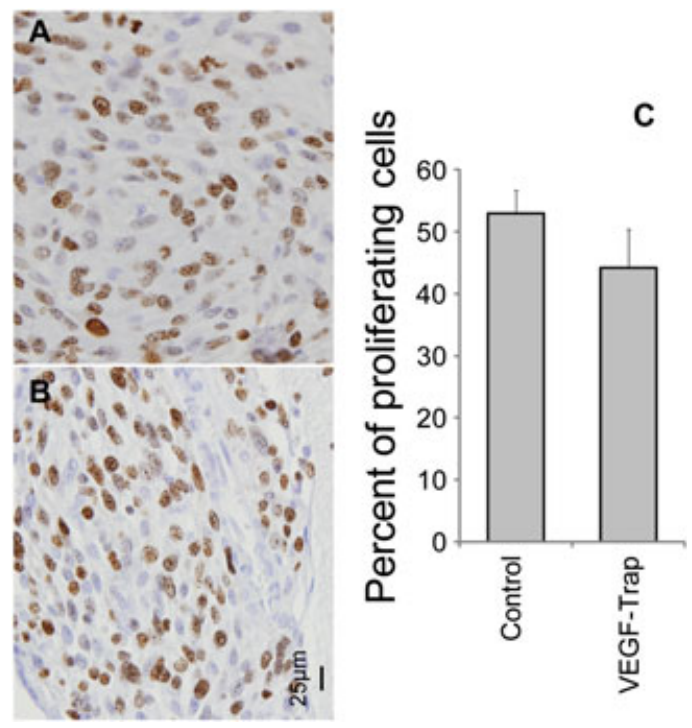

Figure 4. The proliferative potential of tumors following treatment with vehicle (A) or VEGF-Trap (B) was determined by Ki-67 staining of samples taken on Day 12. Positively identified nuclei were counted in randomly selected fields. Representative micrographs for each group are shown. The quantification of the nuclei for each treatment group in 3-6 randomly selected fields per subject (C). Insignificant differences in Ki-67 positive nuclei were observed between treatment groups $(p=0.25)$. Bar plots are presented as the mean number of nuclei and SEM. Images were acquired at $40 \times$ magnification.
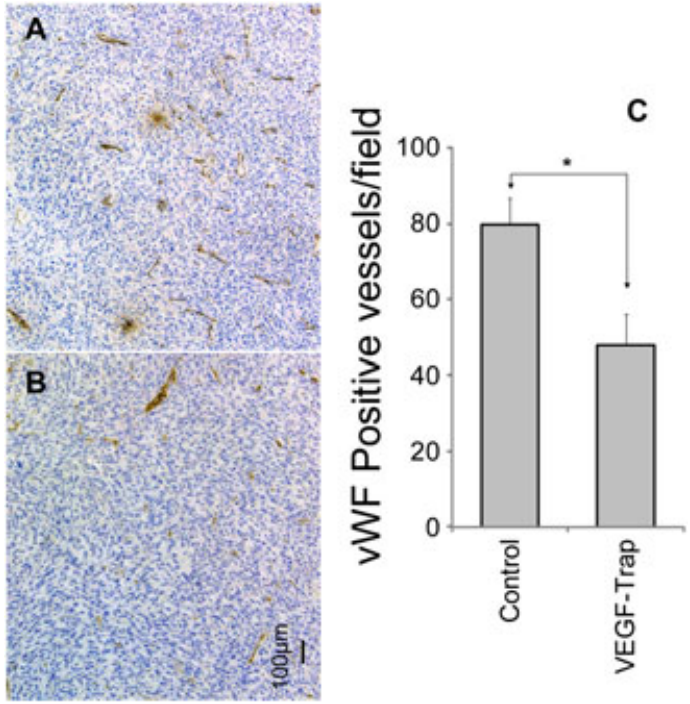

Figure 5. Tumor vasculature following treatment with vehicle $(A)$ or VEGF-Trap (B) was determined by Von Willebrand factor staining of samples taken on Day 12. Positively stained vessels were counted in randomly selected fields. Representative micrographs for each group are shown. The quantification of the vessels for each treatment group in 2-3 randomly selected fields per subject (C). A significant decrease in the number of vessels occurred in VEGF-Trap treated animals $(p=0.011)$. Bar plots are presented as the mean number of nuclei and SEM. Images were acquired at $10 \times$ magnification.

inhibition did not result in a reduction in tumor cellularity as depicted by visual inspection of tumor nuclear staining as well as lack of significant ApopTag staining in the tumor (Fig. 6).

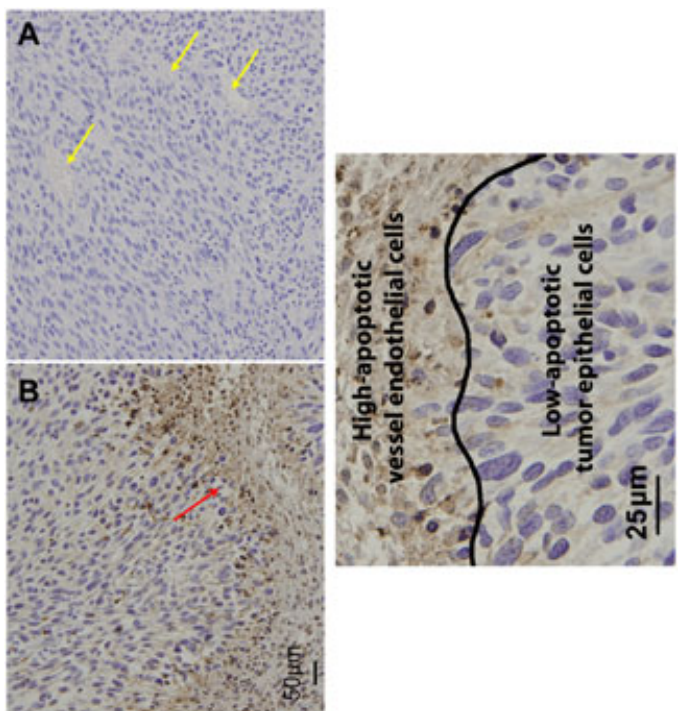

Figure 6. Apoptosis and tumor cellularity following treatment with vehicle (A) or VEGF-Trap (B) was determined by ApopTag staining of samples taken on Day 12, superimposed on H\&E. Extent of apoptosis and tumor cellularity were assessed by visual inspection. Representative micrographs for each group are shown. Treatment by VEGF-Trap resulted in massive apoptotic events in the tumor vasculature but negligible change in tumor cellularity when compared to vehicle-treated tumors. Healthy and apoptotic vessels are indicated by yellow and red arrows, respectively. A closer representative VEGF-Trap treated sample is shown in $\mathrm{C}$, highlighting the border between tumor epithelial and vessel endothelial cells. Images were acquired at $20 \times(A \& B)$ or $40 \times(C)$ magnification.

\section{DISCUSSION}

The development of molecular targeted cancer therapies represents an area of intense investigation. Consequently, a number of clinical trials are underway encompassing a diverse array of targets and agents. However, the primary endpoint used for assessing therapeutic response continues to be gross tumor volumetric change. This endpoint may not be the best choice for measuring the effectiveness of those molecularly targeted agents that do not uniformly elicit a significant reduction in tumor volume (37-39). Therefore, there is an impetus to explore non-invasive quantitative imaging modalities, such as MRI and positron emission tomography, for their potential to provide non-invasive biomarkers of treatment response. In fact, DCEMRI and DW-MRI metrics have shown significant promise as biomarkers of early cancer therapeutic response $(40,41)$. The goal of this study was to evaluate DCE-MRI and DW-MRI for their ability to detect and quantify the therapeutic response of gliomabearing animals treated with VEGF-Trap.

VEGF-Trap is extremely effective in binding VEGF and PGF $(15,18)$. The extent of VEGF-Trap binding to endogenous VEGF was virtually complete following two weeks of treatment (Table 1). This strong binding affinity to endogenous VEGF was clearly evident early in our MRI measurements. Vascular kinetic rates and blood plasma volume fraction as measured by DCE-MRI were highly sensitive to VEGF-Trap treatment. Subsequent to the first treatment, both $K^{\text {trans }}$ and $v_{p}$ diminished by approximately $30 \%$ and $60 \%$, respectively, suggesting an extremely rapid response of tumor vasculature to VEGF-Trap. These vascular changes are reflected in our VWF and ApopTag histological stains (Figs 5 and 6), which show a lower number of VWF-positive-stained blood 
vessels and greater ApopTag staining of vessel endothelial cells in treated animals. These trends agree with those observed in both preclinical and clinical investigations of tumor response to antiangiogenic and antivascular agents as measured by DCE-MRI $(8,41)$.

An increase in tumor water mobility as determined by ADC measurements has been associated with a reduction of tumor cellularity as a consequence of massive cell kill (27). Treatment by VEGF-Trap elicited no such response in ADC values, suggesting no substantial decrease in tumor cellularity due to cell death. Staining by H\&E and ApopTag corroborated what was observed by $A D C$. In fact, ADC values decreased significantly following VEGF-Trap treatment with a significant drop observed as early as one day post-treatment. Reiger and colleagues monitored changes in DSC-MRI and DW-MRI quantitative metrics during Bevacizumab treatment in glioma patients, observing a similar trend in ADC (41). In their clinical study, a drop in tumor blood volume as well as water mobility within the tumor was reported by 8 weeks post-treatment initiation. The reduction of ADC was attributed to pathological changes in the tumor, which may result in a decrease in extracellular water content and narrowing of extracellular space due to treatment-induced hypoxia. This would result in an increase in tumor cellularity per unit volume, which is inversely related to ADC values.

Numerous models for assessing pharmacokinetic qualities in vivo with varying assumptions have been proposed. In this study we used an established model that assumes the fastexchange limit to analyze our DCE-MRI data. This technique has been well-documented but recent studies have provided more robust models including the shutter-speed approach developed by Yankeelov and colleagues in 2003 (34). This model has shown greater accuracy in quantifying permeability kinetics but has only recently seen more extensive application. Although more accurate, the sensitivity of the newer models to therapeutic response have yet to be tested against established pharmacokinetic models. Such an analysis is beyond the scope of this study.

The impairment of VEGF signaling activity in the $9 \mathrm{~L}$ glioma model resulted in apoptosis of the vascular endothelial cell population, which likely contributed to the overall reduction in tumor vessel numbers. The observed decrease in tumor vessel density is also in agreement with previously reported results of the use of antivascular agents on gliomas (42-45). However, in these previous studies, apoptosis of endothelial cells was not reported following VEGF-Trap treatment. Erber and colleagues showed that targeting VEGFR-2 and PDGFR- $\beta$ signaling using the tyrosine kinase inhibitor SU6668 in a C6 rat tumor model resulted in endothelial cell apoptosis and reduced tumor growth rate (46). This is in agreement with our results, which show a similar reduced growth rate as reflected in our volume measurements as well as Ki-67 staining (Fig. 1B and 4) that show a slightly diminished fraction of proliferating cells.

In conclusion, this study supports the utility of DCE- and DW$M R I$ in monitoring the effectiveness of angiogenesis-targeted cancer therapy, in this case response to VEGF-Trap. The ability to track therapeutic effectiveness with non-invasive imaging biomarkers is especially important for gliomas because biopsies during the course of treatment are not an option (unlike most other histotypes that are amenable to pharmacodynamic biomarkers). The use of these MRI modalities is especially compelling as angiogenesis targets are prominently being tested in the glioma population. Besides Aflibercept (VEGF-Trap), there are a number of other targeted agents in current clinical trials for treating glioma patients, e.g. Zactima (vandetanib, ClinicalTrials. gov ID: NCT00272350), cediranib (AZD2171) (35), ramucirumab (ClinicalTrials.gov ID: NCT00895180), BIBF1120 (ClinicalTrials.gov ID: NCT01380782), as well as numerous bevacizumab (Avastin; ClinicalTrials.gov ID: NCT00782756) (47-49) combination trials. Studies are underway to extend this proof-of-principle to the study of the broader angiogenesis portfolio to build a compelling case for clinical trial incorporation. Overall, MRI biomarkers have significant potential for not only monitoring treatment effects but also for optimization of drug dose and schedules.

\section{Acknowledgement}

This work was supported by the National Institutes of Health Research Grants P01CA85878 and P50CA93990. VEGF-Trap was kindly provided by Regeneron, Inc.

\section{REFERENCES}

1. Stupp R, Mason WP, van den Bent MJ, Weller M, Fisher B, Taphoorn MJ, Belanger K, Brandes AA, Marosi C, Bogdahn U, Curschmann J, Janzer RC, Ludwin SK, Gorlia T, Allgeier A, Lacombe D, Cairncross JG, Eisenhauer E, Mirimanoff RO. Radiotherapy plus concomitant and adjuvant temozolomide for glioblastoma. N. Engl. J. Med. 2005; 352(10): 987-996.

2. Thaker NG, Pollack IF. Molecularly targeted therapies for malignant glioma: rationale for combinatorial strategies. Expert. Rev. Neurother. 2009; 9(12): 1815-1836.

3. Yamanaka R, Saya H. Molecularly targeted therapies for glioma. Ann. Neurol. 2009; 66(6): 717-729.

4. Ferrara N, Kerbel RS. Angiogenesis as a therapeutic target. Nature 2005; 438(7070): 967-974.

5. Gasparini G, Longo R, Toi M, Ferrara N. Angiogenic inhibitors: a new therapeutic strategy in oncology. Nat. Clin. Pract. Oncol. 2005; 2(11): 562-577.

6. Raizer JJ, Grimm S, Chamberlain MC, Nicholas MK, Chandler JP, Muro K, Dubner S, Rademaker AW, Renfrow J, Bredel M. A phase 2 trial of single-agent bevacizumab given in an every-3-week schedule for patients with recurrent high-grade gliomas. Cancer 2010; 116(22): 5297-5305.

7. Schmidt NO, Westphal M, Hagel C, Ergun S, Stavrou D, Rosen EM, Lamszus K. Levels of vascular endothelial growth factor, hepatocyte growth factor/scatter factor and basic fibroblast growth factor in human gliomas and their relation to angiogenesis. Int. J. Cancer 1999; 84(1): 10-18.

8. Bauerle T, Bartling S, Berger M, Schmitt-Graff A, Hilbig H, Kauczor HU, Delorme $S$, Kiessling F. Imaging anti-angiogenic treatment response with DCE-VCT, DCE-MRI and DWI in an animal model of breast cancer bone metastasis. Eur. J. Radiol. 2010; 73(2): 280-287.

9. Byrne AT, Ross L, Holash J, Nakanishi M, Hu L, Hofmann JI, Yancopoulos GD, Jaffe RB. Vascular endothelial growth factor-trap decreases tumor burden, inhibits ascites, and causes dramatic vascular remodeling in an ovarian cancer model. Clin. Cancer Res. 2003; 9(15): 5721-5728.

10. Inai $T$, Mancuso $M$, Hashizume $H$, Baffert $F$, Haskell $A$, Baluk $P$, Hu-Lowe DD, Shalinsky DR, Thurston G, Yancopoulos GD, McDonald DM. Inhibition of vascular endothelial growth factor (VEGF) signaling in cancer causes loss of endothelial fenestrations, regression of tumor vessels, and appearance of basement membrane ghosts. Am. J. Pathol. 2004; 165(1): 35-52.

11. Kadenhe-Chiweshe A, Papa J, McCrudden KW, Frischer J, Bae JO, Huang J, Fisher J, Lefkowitch JH, Feirt N, Rudge J, Holash J, Yancopoulos GD, Kandel JJ, Yamashiro DJ. Sustained VEGF blockade results in microenvironmental sequestration of VEGF by tumors and persistent VEGF receptor-2 activation. Mol. Cancer Res. 2008; 6(1): 1-9.

12. Maxwell RJ, Wilson J, Prise VE, Vojnovic B, Rustin GJ, Lodge MA, Tozer GM. Evaluation of the anti-vascular effects of combretastatin in rodent tumours by dynamic contrast enhanced MRI. NMR Biomed. 2002; 15(2): 89-98.

13. Raatschen HJ, Simon GH, Fu Y, Sennino B, Shames DM, Wendland MF, McDonald DM, Brasch RC. Vascular permeability during antiangiogenesis treatment: MR imaging assay results as biomarker for subsequent tumor growth in rats. Radiology 2008; 247(2): 391-399. 
14. Riely GJ, Miller VA. Vascular endothelial growth factor trap in non small cell lung cancer. Clin. Cancer Res. 2007; 13 (15 Pt 2): s4623-s4627.

15. Huang J, Frischer JS, Serur A, Kadenhe A, Yokoi A, McCrudden KW, New T, O'Toole K, Zabski S, Rudge JS, Holash J, Yancopoulos GD, Yamashiro DJ, Kandel JJ. Regression of established tumors and metastases by potent vascular endothelial growth factor blockade. Proc. Natl Acad. Sci. USA 2003; 100(13): 7785-7790.

16. Baffert F, Le T, Sennino B, Thurston G, Kuo CJ, Hu-Lowe D, McDonald DM. Cellular changes in normal blood capillaries undergoing regression after inhibition of VEGF signaling. Am. J. Physiol. 2006; 290(2): H547-H559.

17. Fricke I, Mirza N, Dupont J, Lockhart C, Jackson A, Lee JH, Sosman JA Gabrilovich DI. Vascular endothelial growth factor-trap overcomes defects in dendritic cell differentiation but does not improve antigenspecific immune responses. Clin. Cancer Res. 2007; 13(16): 4840-4848.

18. Holash J, Davis S, Papadopoulos N, Croll SD, Ho L, Russell M, Boland $P$, Leidich R, Hylton D, Burova E, loffe E, Huang T, Radziejewski C, Bailey K, Fandl JP, Daly T, Wiegand SJ, Yancopoulos GD, Rudge JS. VEGF-Trap: a VEGF blocker with potent antitumor effects. Proc. Natl Acad. Sci. USA 2002; 99(17): 11 393-11 398.

19. Rudge JS, Holash J, Hylton D, Russell M, Jiang S, Leidich R, Papadopoulos N, Pyles EA, Torri A, Wiegand SJ, Thurston G, Stahl N, Yancopoulos GD. Inaugural Article: VEGF Trap complex formation measures production rates of VEGF, providing a biomarker for predicting efficacious angiogenic blockade. Proc. Natl Acad. Sci. USA 2007; 104(47): 18 363-18 370.

20. Kim ES, Serur A, Huang J, Manley CA, McCrudden KW, Frischer JS, Soffer SZ, Ring L, New T, Zabski S, Rudge JS, Holash J, Yancopoulos GD, Kandel JJ, Yamashiro DJ. Potent VEGF blockade causes regression of coopted vessels in a model of neuroblastoma. Proc. Natl Acad. Sci. USA 2002; 99(17): 11 399-11 404.

21. Bergers G, Benjamin LE. Tumorigenesis and the angiogenic switch. Nature Rev. 2003; 3(6): 401-410.

22. Hanahan D, Weinberg RA. The hallmarks of cancer. Cell 2000; 100(1): 57-70.

23. Yankeelov TE, Gore JC. Dynamic Contrast Enhanced Magnetic Resonance Imaging in Oncology: Theory, Data Acquisition, Analysis, and Examples. Curr. Med. Imaging Rev. 2009; 3(2): 91-107.

24. Kety SS. The theory and applications of the exchange of inert gas at the lungs and tissues. Pharmacol. Rev. 1951; 3(1): 1-41.

25. Kovar DA, Lewis M, Karczmar GS. A new method for imaging perfusion and contrast extraction fraction: input functions derived from reference tissues. J. Magn. Reson. Imaging 1998; 8(5): 1126-1134.

26. Tofts PS. Modeling tracer kinetics in dynamic Gd-DTPA MR imaging. J. Magn. Reson. Imaging 1997; 7(1): 91-101.

27. Chenevert TL, Stegman LD, Taylor JM, Robertson PL, Greenberg HS, Rehemtulla A, Ross BD. Diffusion magnetic resonance imaging: an early surrogate marker of therapeutic efficacy in brain tumors. J. Natl Cancer Inst. 2000; 92(24): 2029-2036.

28. Chenevert TL, Sundgren PC, Ross BD. Diffusion imaging: insight to cell status and cytoarchitecture. Neuroimag. Clin. N. Am. 2006; 16 (4): 619-632, viii-ix.

29. Galban S, Brisset JC, Rehemtulla A, Chenevert TL, Ross BD, Galban CJ. Diffusion-weighted MRI for assessment of early cancer treatment response. Curr. Pharm. Biotechnol. 2010; 11(6): 701-708.

30. Hamstra DA, Rehemtulla A, Ross BD. Diffusion magnetic resonance imaging: a biomarker for treatment response in oncology. J. Clin. Oncol. 2007; 25(26): 4104-4109.

31. Ellingson BM, Malkin MG, Rand SD, Connelly JM, Quinsey C, LaViolette PS, Bedekar DP, Schmainda KM. Validation of functional diffusion maps (fDMs) as a biomarker for human glioma cellularity. J. Magn. Reson. Imaging 2010; 31(3): 538-548.

32. Moffat BA, Hall DE, Stojanovska J, McConville PJ, Moody JB, Chenevert TL, Rehemtulla A, Ross BD. Diffusion imaging for evaluation of tumor therapies in preclinical animal models. Magn. Reson. Mater. Phy. 2004; 17(3-6): 249-259.

33. Ross BD, Zhao YJ, Neal ER, Stegman LD, Ercolani M, Ben-Yoseph O, Chenevert TL. Contributions of cell kill and posttreatment tumor growth rates to the repopulation of intracerebral $9 \mathrm{~L}$ tumors after chemotherapy: an MRI study. Proc. Natl Acad. Sci. USA 1998; 95(12): 7012-7017.

34. Yankeelov TE, Rooney WD, Li X, Springer CS, Jr. Variation of the relaxographic "shutter-speed" for transcytolemmal water exchange affects the CR bolus-tracking curve shape. Magn. Reson. Med. 2003; 50(6): 1151-1169.
35. Batchelor TT, Sorensen AG, di Tomaso E, Zhang WT, Duda DG, Cohen KS, Kozak KR, Cahill DP, Chen PJ, Zhu M, Ancukiewicz M, Mrugala MM, Plotkin S, Drappatz J, Louis DN, Ivy P, Scadden DT, Benner T, Loeffler JS, Wen PY, Jain RK. AZD2171, a pan-VEGF receptor tyrosine kinase inhibitor, normalizes tumor vasculature and alleviates edema in glioblastoma patients. Cancer Cell 2007; 11(1): 83-95.

36. Bocci G, Man S, Green SK, Francia G, Ebos JM, du Manoir JM, Weinerman $A$, Emmenegger $U, M a ~ L$, Thorpe $P$, Davidoff $A$, Huber J, Hicklin DJ, Kerbel RS. Increased plasma vascular endothelial growth factor (VEGF) as a surrogate marker for optimal therapeutic dosing of VEGF receptor-2 monoclonal antibodies. Cancer Res. 2004; 64(18): 6616-6625.

37. Choi H, Charnsangavej C, de Castro Faria S, Tamm EP, Benjamin RS, Johnson MM, Macapinlac HA, Podoloff DA. CT evaluation of the response of gastrointestinal stromal tumors after imatinib mesylate treatment: a quantitative analysis correlated with FDG PET findings. Am. J. Roentgenol. 2004; 183(6): 1619-1628.

38. Jaffe CC. Measures of response: RECIST, WHO, and new alternatives. J. Clin. Oncol. 2006; 24(20): 3245-3251.

39. Strumberg $D$, Richly $H$, Hilger RA, Schleucher $N$, Korfee $S$, Tewes $M$, Faghih M, Brendel E, Voliotis D, Haase CG, Schwartz B, Awada A, Voigtmann R, Scheulen ME, Seeber S. Phase I clinical and pharmacokinetic study of the Novel Raf kinase and vascular endothelial growth factor receptor inhibitor BAY 43-9006 in patients with advanced refractory solid tumors. J. Clin. Oncol. 2005; 23(5): 965-972.

40. Hamstra DA, Galban CJ, Meyer CR, Johnson TD, Sundgren PC, Tsien C, Lawrence TS, Junck L, Ross DJ, Rehemtulla A, Ross BD, Chenevert TL. Functional diffusion map as an early imaging biomarker for high-grade glioma: correlation with conventional radiologic response and overall survival. J. Clin. Oncol. 2008; 26(20): 3387-3394.

41. Sorensen AG, Batchelor TT, Zhang WT, Chen PJ, Yeo P, Wang M, Jennings D, Wen PY, Lahdenranta J, Ancukiewicz M, di Tomaso E, Duda DG, Jain RK. A "vascular normalization index" as potential mechanistic biomarker to predict survival after a single dose of cediranib in recurrent glioblastoma patients. Cancer Res. 2009; 69(13): 5296-5300.

42. Wachsberger PR, Burd R, Cardi C, Thakur M, Daskalakis C, Holash J, Yancopoulos GD, Dicker AP. VEGF trap in combination with radiotherapy improves tumor control in u87 glioblastoma. Int. J. Rad. Oncol. 2007; 67(5): 1526-1537.

43. Gomez-Manzano C, Holash J, Fueyo J, Xu J, Conrad CA, Aldape KD, de Groot JF, Bekele BN, Yung WK. VEGF Trap induces antiglioma effect at different stages of disease. Neuro-Oncology 2008; 10(6): 940-945.

44. Kunkel $P$, Ulbricht $U$, Bohlen $P$, Brockmann MA, Fillbrandt $R$, Stavrou $D$, Westphal M, Lamszus K. Inhibition of glioma angiogenesis and growth in vivo by systemic treatment with a monoclonal antibody against vascular endothelial growth factor receptor-2. Cancer Res. 2001; 61(18): 6624-6628.

45. Rubenstein JL, Kim J, Ozawa T, Zhang M, Westphal M, Deen DF, Shuman MA. Anti-VEGF antibody treatment of glioblastoma prolongs survival but results in increased vascular cooption. Neoplasia 2000; 2(4): 306-314.

46. Erber R, Thurnher A, Katsen AD, Groth $G$, Kerger $H$, Hammes HP, Menger MD, Ullrich A, Vajkoczy P. Combined inhibition of VEGF and PDGF signaling enforces tumor vessel regression by interfering with pericyte-mediated endothelial cell survival mechanisms. J. Fed. Am. Society Exp. Biol 2004; 18(2): 338-340.

47. Drappatz J, Lee EQ, Hammond S, Grimm SA, Norden AD, Beroukhim R, Gerard M, Schiff D, Chi AS, Batchelor TT, Doherty LM, Ciampa AS, Lafrankie DC, Ruland S, Snodgrass SM, Raizer JJ, Wen PY. Phase I study of panobinostat in combination with bevacizumab for recurrent high-grade glioma. J. Neurooncol. 2011. DOI: 10.1007/ s11060-011-0717-z

48. Vredenburgh JJ, Desjardins A, Reardon DA, Peters KB, Herndon JE, 2nd, Marcello J, Kirkpatrick JP, Sampson JH, Bailey L, Threatt S, Friedman $\mathrm{AH}$, Bigner DD, Friedman HS. The addition of bevacizumab to standard radiation therapy and temozolomide followed by bevacizumab, temozolomide, and irinotecan for newly diagnosed glioblastoma. Clin. Cancer Res. 2011; 17(12): 4119-4124.

49. Zhang W, Fulci G, Buhrman JS, Stemmer-Rachamimov AO, Chen JW, Wojtkiewicz GR, Weissleder R, Rabkin SD, Martuza RL. Bevacizumab with angiostatin-armed oHSV increases antiangiogenesis and decreases Bevacizumab-induced invasion in U87 glioma. Mol. Ther. 2011. DOI: 10.1038/mt.2011.187 